\title{
Uma análise crítica da aplicação do método Janela do Cliente na avaliação da qualidade do Restaurante Universitário
}

A critical analysis of the application of Customer Window method in quality assessment of the University Restaurant

\author{
Angelica Peripolli', Bruna Gregory Palm", Caroline Pafiadache da Silva Piekala"', \\ Laís Helen Loose" ${ }^{\mathrm{V}}$, Rafael Piaiav", Vinícius Teodoro Scher"v", \\ Fernando de Jesus Moreira Junior VII
}

\begin{abstract}
RESUMO
O método da Janela do Cliente tem por principal função medir o nível de importância e o grau de satisfação em relação aos produtos ou serviços prestados pela empresa, sendo uma ferramenta capaz de identificar os pontos falhos e as potencialidades da empresa. O objetivo desse artigo é apresentar uma análise crítica da aplicação do método Janela do Cliente na avaliação da qualidade do Restaurante Universitário da Universidade Federal de Santa Maria - RS (UFSM). Participaram da pesquisa, 1.855 usuários, entre alunos, docentes e técnicos administrativos. Os resultados mostraram que, nas três unidades do Restaurante Universitário, a maioria dos itens avaliados encontra-se no Quadrante A da Janela do Cliente, denominado "força competitiva", ou seja, a maioria dos itens é importante para o usuário e são atendidos de forma satisfatória pelos restaurantes. No entanto, alguns itens encontramse no Quadrante D da Janela do Cliente, denominado "vulnerabilidade competitiva", sendo esses itens importantes para o usuário, porém não estão sendo atendidos de forma adequada. Os resultados dessa pesquisa foram encaminhados à administração do Restaurante Universitário, onde poderão gerar subsídios para formulação de propostas que visam melhorias (mudanças ou adaptações) nos atributos avaliados.
\end{abstract}

Palavras-chave: Restaurante Universitário; Janela do Cliente; Pesquisa de Satisfação.

\begin{abstract}
The Costumer Window method has the main function to measure the level of importance and the degree of satisfaction with the products or services provided by the company, being a tool able to identify the weak points and powers of the company. The purpose of this paper is to present a critical analysis of the application of the method the client window in quality evaluation of University Restaurant, from Federal University of Santa Maria - RS (UFSM). The research involved 1,855 users, including students, teachers and administrative staff. The results showed that in the three units of the University Restaurant, most of the items evaluated is in Quadrant A of the Costumer Window, called "competitive force", ie, most of the items is important to the user and are attended of satisfactorily by restaurants. However, some items are in Quadrant D of the Costumer Window, called "competitive vulnerability", these items are important to the user but they are not being attended adequately. The results of this survey were forwarded to the administration of University Restaurant, which can generate subsides for the formulation of proposals to improvements (changes or adaptations) in the assessed attributes.
\end{abstract}

Keywords: University Restaurant; Customer Window; Satisfaction survey. 


\section{INTRODUÇÃO}

Num mundo onde as pessoas estão com seu tempo cada vez mais limitado diante das inúmeras responsabilidades adquiridas, o antigo hábito de fazer todas as refeições em casa vem se tornando algo cada vez mais raro. Nesse sentido, Passador et al (2006) afirmam que a busca pela praticidade aliada à falta de tempo e a distância entre o local de trabalho e a residência fez com que as pessoas mudassem seus hábitos alimentares, de maneira que muitas destas passaram a realizar as refeições fora de casa. Dentro desse contexto, está incluída até a comunidade acadêmica (estudantes, docentes, servidores técnicos administrativos) que, por diversos motivos, acaba optando por fazer suas refeições na própria universidade. Os restaurantes universitários (RU's) têm sua importância fundamentada sob três aspectos básicos distintos: o prazer, no que se refere à alimentação como uma satisfação, um desejo pessoal; a praticidade, que pode ser aliada tanto ao desejo da refeição, como uma necessidade; e por último, a saúde, geralmente ligada à uma necessidade específica do comensal de realizar esta refeição.

O serviço de alimentação em um campus é completamente diferente dos outros tipos de restaurantes, ou seja, em restaurantes tradicionais os clientes vão até lá por causa do estabelecimento em si, onde comer é o propósito principal. Por outro lado, num campus universitário, os usuários estão lá pelas aulas ou trabalho e comer no RU é uma necessidade básica motivada pelas circunstâncias. Nesse sentido, poucas pessoas vão para o campus apenas para comer (KLASSEN; KUMAR; TRYBUS, 2005).

O Restaurante Universitário convive diariamente com o desafio de manter, a um baixo custo um atendimento de qualidade, além de prover uma refeição saudável, atendendo às necessidades nutricionais dos usuários, oferecendo um cardápio qualitativo e quantitativamente variado e equilibrado. Sua função é atender os funcionários e os alunos da universidade durante o cumprimento de suas funções na universidade (MAIA, 2008; OLIVEIRA; ALVES, 2008; BRANDÃO; GIOVANONI, 2011).

Segundo Fonaprace (1997), os RU's, no contexto das Instituições Públicas de Ensino Superior, representam não só a democratização do espaço universitário, mas uma necessidade para o melhor funcionamento dessas Instituições, inclusive sendo 
responsáveis diretos, em algumas delas, pela redução dos índices de evasão escolar. Existem vários trabalhos científicos que comprovam que os Restaurantes Universitários contribuem na melhora não só o rendimento acadêmico, como também as condições de vida das pessoas.

A alimentação é um dos fatores fundamentais para a saúde e bem-estar do homem, fornecendo nutrientes necessários para as várias funções do organismo. É necessário salientar que esta contribuição da alimentação ocorre por meio do consumo de alimentos, sendo que o indivíduo sente necessidade de sabor, textura, aroma e cor agradáveis (PASTORE; KLIEMANN; FERNANDES; OLIVEIRA; CORSO; PROENÇA, 2009). Conforme Proença (1999), as alterações ambientais têm feito com que o setor de alimentação coletiva experimentasse mudanças significativas nos aspectos de qualidade, voltados tanto às questões de higiene e sanidade dos alimentos e preparações, quanto ao atendimento de normas que regem o preparo e distribuição de alimentos. Na visão de Teixeira, Millet, Carvalho e Biscontini (2001), para atender as condições de bem-estar em um refeitório, é necessário um planejamento físico, no qual, deve ser de natureza abrangente e envolver dimensionamento da área, ambiência, composição e ergonomia, que favoreça e realce os fatores ambientais.

O objetivo desse trabalho é apresentar uma análise crítica da aplicação da ferramenta conhecida como Janela do Cliente na avaliação da qualidade do Restaurante Universitário da Universidade Federal de Santa Maria (UFSM).

\section{JANELA DO CLIENTE}

A satisfação dos clientes é a maneira mais eficiente de se avaliar o desempenho de uma organização. Segundo McDaniel e Gates (2006), a pesquisa de Marketing é a principal ferramenta para obter informações oportunas para a tomada de decisão e para buscar novas oportunidades de mercado, pois permite fornecer aos tomadores de decisões dados sobre o diagnóstico atual e oferecer ideias sobre as mudanças necessárias. Uma pesquisa de satisfação proporciona vários benefícios como obter uma percepção mais positiva dos clientes com relação à empresa; informações atuais e relevantes quanto às necessidades dos clientes; cria relação de lealdade por parte dos clientes e dá aos mesmos, confiança devido a aproximação com a empresa, nesse 
caso, o Restaurante Universitário. A pesquisa de satisfação também tem como objetivos criar padrões mais adequados de atendimento aos clientes, aumentar o índice de retenção dos mesmos, maximizar a sua satisfação e anular o índice de insatisfação (ROSSI; SLONGO, 1998).

Já a análise da importância permite uma visão priorizada sobre quais atributos do serviço devem ser alterados e ajuda a entender o desempenho relativo de determinado critério em função da importância dada pelo consumidor a esse critério (MARTILLA; JAMES, 1977). Aaker, Kumar e Day (2001) afirmam que a mensuração da importância dos atributos de produtos e serviços pelos clientes pode representar uma fonte potencial para ser utilizada de três maneiras principais: na segmentação dos clientes e ajuste da oferta de serviços das empresas; no controle das interações de serviço, identificando os atributos críticos de serviço para então, estabelecer padrões satisfatórios de entrega de serviço; na mensuração da qualidade e satisfação de serviço percebido. Segundo Haley (1985), o conhecimento dos benefícios, necessidades e desejos do cliente propicia uma melhor ação de marketing do que o conhecimento de variáveis demográficas ou socioeconômicas.

A ferramenta de pesquisa Janela do Cliente tem por principal função medir o nível de importância e o grau de satisfação em relação aos produtos ou serviços prestados por determinada empresa (MATTOS, 2008). É uma metodologia de pesquisa que serve para medir o grau de satisfação dos clientes tanto externos, quanto internos, em relação às características de qualidade dos produtos e serviços fornecidos (ASSESSORIA DE PESQUISA E DESENVOLVIMENTO GERENCIAL, 1994). Esta ferramenta é um resultado obtido a partir da aplicação de uma pesquisa de mercado através de um questionário utilizado para medir o nível de importância e o grau de satisfação sobre os mesmos atributos. "Janela do Cliente" foi aplicada inicialmente no ano de 1987, pela Arbor Incorporation, com o nome de Customer Window, inspirada no artigo Market Research the Japonese Way da Harvard Business Review (SCHLENDER, 2013). No ano de 1989, após algumas modificações, essa mesma ferramenta foi novamente apresentada no congresso da American Society for Quality Control, onde estiveram presentes os funcionários do Grupo Rhodia, primeira empresa brasileira a utilizar esta ferramenta, 
a partir da década de 90. A Rhodia passou a aplicar a Janela do Cliente no desenvolvimento de diversos projetos, inclusive para medir a satisfação dos clientes internos (CÁCERES, 1994). Muitos autores mencionam que os autores Albrecht e Bradford (1992) definem o termo "Janela do Cliente", no entanto, esse termo sequer é mencionado. O que é conhecido como "Janela do Cliente", estes autores chamam de "matriz de atributos de serviço", que é um gráfico que cruza duas variáveis. Conforme Rosa (2011), uma variável (Y) avalia a importância percebida sob determinado atributo, ou seja, a importância que o cliente atribui à esse atributo; e a outra variável $(X)$ avalia o nível de desempenho (ou grau de satisfação) em relação a este mesmo atributo, ou seja, aquilo que o cliente sente sobre o mesmo atributo. Segundo Notari (2002), o nome "Janela do Cliente" atribuído a essa ferramenta se deve ao fato que a aparência do gráfico resultante lembra uma janela dividida em quatro quadrantes.

Utiliza-se uma matriz de dupla entrada (importância e satisfação) que possibilita mensurar cada atributo de acordo com estas duas variáveis, por meio de uma visualização gráfica (LUDWING, 2008), assim, é possível identificar os pontos falhos e as potências da empresa em estudo (PORTAL, 2012). A Janela do Cliente é um diagrama de dispersão entre os valores atribuídos à satisfação (eixo $\mathrm{X}$ ) e à importância (eixo Y) dos atributos avaliados. A Figura 1 apresenta a divisão da Janela do Cliente em quatro quadrantes interpretáveis.

Figura 1 - A Janela do Cliente e os quatro quadrantes

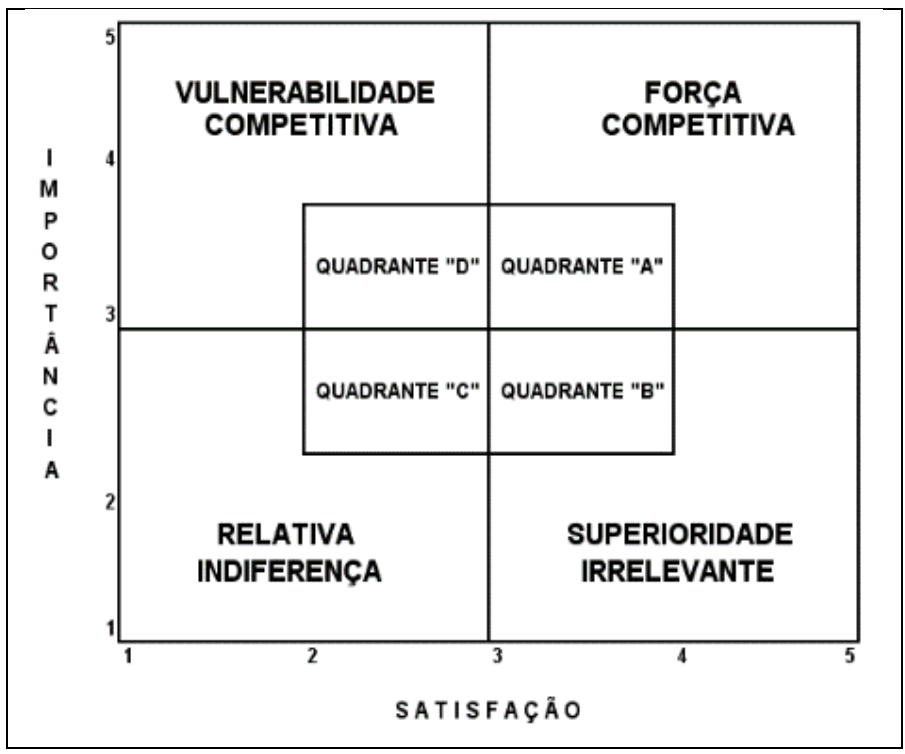

Fonte: Mattos (2008). 
Observa-se que na parte vertical da matriz (eixo Y) encontra-se o nível de importância, e na parte horizontal encontra-se o grau de satisfação (eixo X), onde estão distribuídos os quadrantes. São esses quatro quadrantes que nos permitem identificar quatro posicionamentos em relação aos atributos. Albrecht e Bradford (1992), Mattos (2008) e Feoli (2006) interpretam os quatro quadrantes da Figura 1 da seguinte forma:

- Quadrante A, denominado "Força Competitiva": os atributos são considerados acima da média, tanto com relação aos níveis de importância, como para os níveis de satisfação. Neste quadrante, o cliente recebe o produto ou serviço da empresa e os valoriza. Este é o quadrante onde as empresas atendem as necessidades e superam as expectativas do cliente com excelência.

- Quadrante B, definido como "Superioridade Irrelevante": os atributos são considerados de pouca importância pelo cliente, mesmo ele estando satisfeito com aquilo que recebe. O interessante é tentar aumentar a percepção do cliente em relação aos atributos localizados neste quadrante, tentando promovê-los do quadrante B para o quadrante A. Este é o quadrante da Janela do Cliente onde as empresas concentram o devido esforço, porém os clientes não dão o devido reconhecimento.

- Quadrante C, identificado como "Relativa Indiferença": refere-se à uma zona onde tanto o nível de importância como o grau de satisfação possuem níveis baixos, segundo a percepção dos clientes. Daí a denominação indiferença, pois o cliente não valoriza esses atributos. Este é o quadrante onde o atributo que está sendo analisado poderia ser importante para o cliente, mas por algum motivo não é. A empresa deve tentar aumentar a percepção de importância e satisfação atribuída aos itens que estão nesse quadrante, para conseguir um diferencial competitivo.

- Quadrante D conhecido como "Vulnerabilidade Competitiva": refere-se à zona que requer maior atenção da empresa. É quando o cliente valoriza o atributo, porém não o recebe de forma satisfatória. Isso, além de gerar clientes insatisfeitos, pode estar resultando na sua perda. São esses atributos que a empresa deverá concentrar todos os seus esforços para modificar o nível de satisfação percebido por seus clientes, possibilitando assim, a obtenção de uma vantagem competitiva frente aos demais concorrentes. 
Souza (2008) resume e ilustra a Janela do Cliente, em termos de satisfação e importância, conforme a Figura 2. Entretanto, o autor nomeia os quadrantes de forma diferente do que foi definido anteriormente.

Figura 2 - A Janela do Cliente e os quatro quadrantes

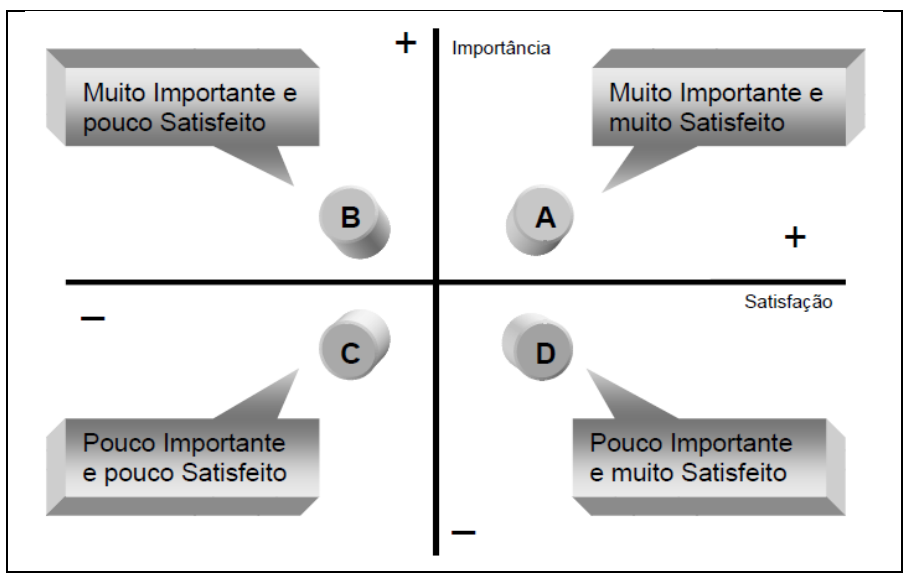

Fonte: Souza (2008).

Ludwing (2008) destaca ainda a presença de uma "zona cinzenta" ou "zona de indiferença", onde a importância do atributo não é nem alta e nem baixa, em relação à média, assim como o nível de satisfação, como ilustra a Figura 3.

Figura 3 - A Janela do Cliente com a zona cinzenta

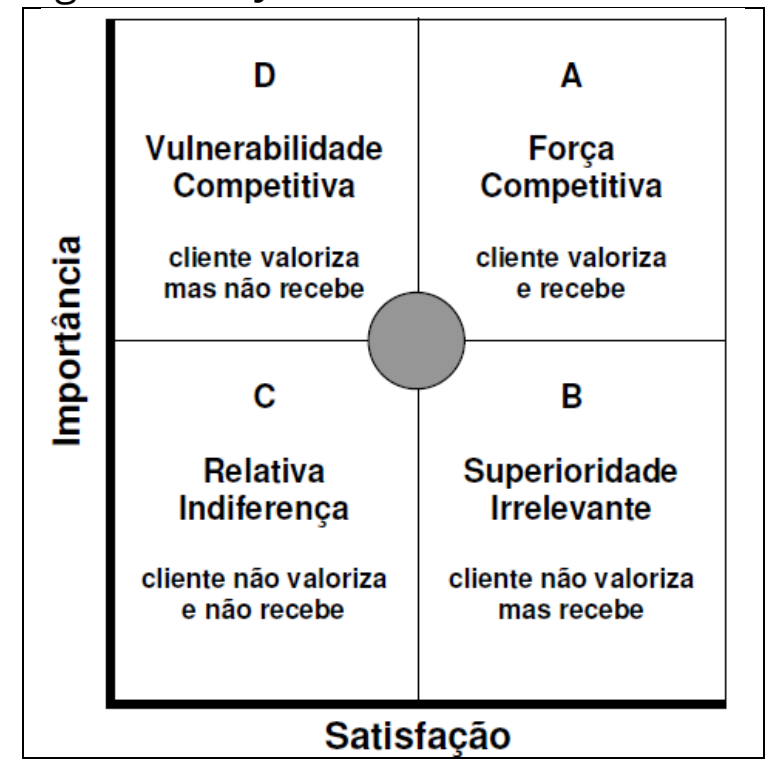

Fonte: Ludwing (2008).

A Figura 4 apresenta a relação entre a importância de um atributo e seu desempenho (ou satisfação), para cada tributo, conforme Albrecht e Bradford (1992). É dessa ilustração básica que surge a ideia da Janela do Cliente. 
Figura 4 - Relação entre a importância de um atributo e seu desempenho

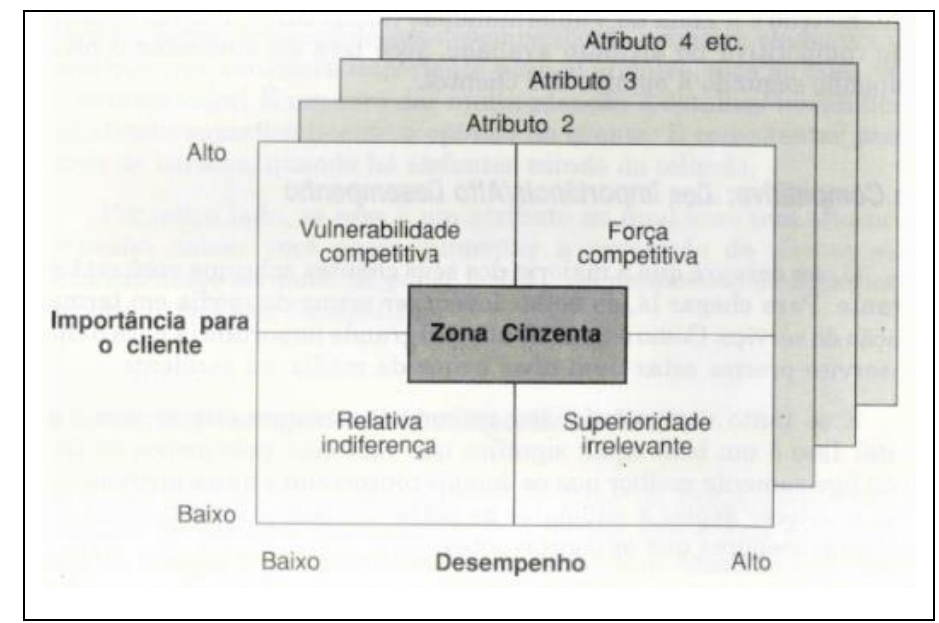

Fonte: Albrecht e Bradford (1992).

Para Albrecht e Bradford (1992), há várias formas de medir a prioridade e a satisfação dos atributos. Uma das possíveis formas é utilizar uma escala likert variando, por exemplo, entre 1 e 5, conforme o grau de importância ou satisfação, onde o valor 3 representaria o valor intermediário, correspondente à "zona cinzenta". Vários autores não consideram a "zona cinzenta" em seus estudos, talvez pela dificuldade em delimitála com exatidão. Para identificar os quatro quadrantes é preciso determinar o cruzamento entre os eixos $\mathrm{X}$ e $\mathrm{Y}$ do gráfico que irá delimitar os quadrantes. Barroso (2007), Feoli (2006), Ludwing (2008), Mattos (2008), Notari (2002), Portal (2012), Silva (2003) e Zabaleta (2011) determinaram o cruzamento segundo a média da satisfação e da importância de todos os atributos, avaliados em uma escala likert de 1 (nada importante ou totalmente insatisfeito) a 5 (muito importante ou totalmente satisfeito). No entanto, esse critério não parece adequado.

Segundo essa abordagem, por mais bem avaliados que os atributos sejam, sempre haverá alguns acima da média e outros abaixo da média, ou seja, sempre haverá atributos em pelo menos dois quadrantes. Por exemplo, se todos os atributos forem bem avaliados, tendo recebido atribuições entre 4 e 5 , tomando por regra a média (que estaria entre 4 e 5) para delimitar os quadrantes, haverá atributos acima da média e abaixo da média, classificando os atributos em pelo menos dois quadrantes, embora todos devessem estar no quadrante A por terem sido bem avaliados. Isso se torna incoerente, pois ocorreria que um atributo que é importante e o cliente está satisfeito, seja classificado como "sem importância" e "insatisfeito". De forma análoga, 
o mesmo ocorre se todos os atributos forem bem avaliados de forma insatisfatória e sem importância. Segundo a abordagem dos autores supracitados, haveriam itens em pelo menos dois quadrantes, embora todos devessem estar no quadrante $\mathrm{C}$ por terem sido mal avaliados. Dessa forma, o método mais coerente parece utilizar os valores médios das escalas, por exemplo, o valor 3 de uma escala likert entre 1 e 5, para delimitar os quadrantes.

\section{METODOLOGIA}

O presente estudo foi realizado através de um survey, ou seja, um questionário quantitativo elaborado com base nos estudos de Carvalho, Amorim e Tavares (2003), Siebeneichler, Wienningkamp, Ruchel, Trombini e Zamberlan (2008) e Garcez, Fachin e Andrade Júnior (2000). O questionário era autoaplicável e foi divulgado na página oficial da Universidade Federal de Santa Maria, em redes sociais e em cartazes fixados em lugares estratégicos do campus universitário onde havia grande circulação de pessoas. Também foi enviado de forma eletrônica ao público-alvo (estudantes de graduação, mestrado e doutorado, técnicos administrativos e docentes), cujos os e-mails estavam cadastrados no SIE - Sistema de Informações para o Ensino da UFSM. O formulário foi elaborado utilizando o aplicativo Google Docs (www.google.com/docs), onde os usuários interessados em participar poderiam responder ao questionário, o que caracteriza uma amostra não-probabilística.

O questionário aplicado estava constituído por três partes, a primeira com abordagens sobre informações do perfil do usuário, a segunda sobre o nível de satisfação do usuário em relação a 41 aspectos relacionados aos restaurantes universitários da UFSM, e a terceira sobre a importância atribuída pelos usuários a esses 41 aspectos. Esse artigo delimita-se a analisar conjuntamente o nível de satisfação dos usuários e importância atribuída pelos mesmos em relação a esses 41 aspectos. Os usuários avaliaram a sua satisfação em relação aos 41 aspectos utilizando a escala likert apresentada na Tabela 1.

Tabela 1 - Escala do nível de satisfação utilizada

\begin{tabular}{ccccc}
\hline Muito insatisfeito Insatisfeito & $\begin{array}{c}\text { Indiferente } \\
\text { (nem satisfeito, nem insatisfeito) }\end{array}$ & Satisfeito Muito satisfeito \\
\hline 1 & 2 & 3 & 4 & 5 \\
\hline
\end{tabular}


Os usuários também atribuíram o grau de importância em relação aos 41 aspectos utilizando a escala likert apresentada na Tabela 2.

Tabela 2 - Escala do grau de importância utilizada

\begin{tabular}{lcccc}
\hline Nada Importante Pouco Importante & Razoavelmente Importante Importante Muito Importante \\
\hline 1 & 2 & 3 & 4 & 5 \\
\hline
\end{tabular}

Um espaço para críticas, elogios e sugestões foi adicionado ao final da pesquisa. As respostas foram separadas por expressões-chave e em seguida foram organizadas em categorias temáticas e apresentadas sob forma de um resumo coletando-se a respectiva ideia central considerada mais significativa, onde os principais resultados foram descritos nesse trabalho. O questionário esteve à disposição para ser respondido pelos frequentadores do Restaurante Universitário entre os dias 16 de janeiro a primeiro de fevereiro de 2013.

As atividades relativas à essa pesquisa foram desenvolvidas nos laboratórios de Estatística situados no Centro de Ciências Naturais e Exatas (CCNE) da Universidade Federal de Santa Maria - UFSM e, para análise dos dados, foram utilizados os softwares estatísticos Statistica 9.0 e Statistical Package for the Social Science - SPSS 17.0, além do Microsoft Excel®. Os dados omissos, ou seja, as perguntas que não obtiveram resposta, não foram avaliadas, logo as análises foram baseadas apenas nas respostas válidas.

O projeto dessa pesquisa foi registrado no Gabinete de Projetos da instituição sob o número 033168 e aprovado pelo Comitê de Ética em Pesquisa da UFSM (CAAE 11217612.6.0000.5346) pelo parecer consubstanciado número 185.858.

\section{RESULTADOS}

Participaram da pesquisa 1.855 usuários do RU da UFSM, sendo esse um número que superou as expectativas da equipe de pesquisa. Desses respondentes, 41,35\% eram homens e $58,65 \%$ mulheres, sendo que no RU do Centro é onde se concentra o maior percentual de mulheres, conforme mostra da Tabela 3. 
Tabela 3 - RU em que almoça X Gênero

\begin{tabular}{lccc}
\hline RU X Gênero & Masculino & Feminino & Total \\
\hline RU Centro & $31,70 \%$ & $68,30 \%$ & $100,00 \%$ \\
RU Campus - Refeitório I & $37,90 \%$ & $62,10 \%$ & $100,00 \%$ \\
RU Campus - Refeitório II & $48,40 \%$ & $51,60 \%$ & $100,00 \%$ \\
\hline Total & $41,30 \%$ & $58,70 \%$ & $100,00 \%$ \\
\hline
\end{tabular}

Vale ressaltar que o restaurante em que o usuário almoça é pré-definido pelo curso onde o mesmo está matriculado, ou seja, o usuário não pode escolher o restaurante em que irá almoçar. Dessa forma, a maioria $(89,9 \%)$ almoça no Campus localizado no bairro Camobi sendo 50,9\% no Refeitório I e 38,9\% no Refeitório II, enquanto que apenas 10,1\% almoça no RU localizado no Centro de Santa Maria, RS.

Em relação ao estado civil, a maioria era solteiro $(85,61 \%)$ seguido dos casados $(12,61 \%)$, e ainda com um pequeno percentual de divorciados $(1,78 \%)$ e viúvos $(0,27 \%)$. Com relação ao vínculo com a UFSM, 92,14\% são alunos, sendo $23,03 \%$ alunos carentes (com benefício socioeconômico) e 69,11\% são alunos sem o benefício, 6,85\% são servidores técnicos administrativos que trabalham na universidade e 1,02\% são docentes que lecionam na instituição. Entre os alunos, 72,61\% cursam a graduação, 17,41\% a Pós-Graduação, 1,24\% fazem um curso técnico e 0,86\% estão no ensino médio, e cerca de 8\% não responderam que curso frequenta na UFSM.

Em geral, a maioria dos usuários declarou que almoça em grupo (76,17\%). Mas ao identificarmos o RU, verificamos que esse comportamento é mais frequente nos RU's do Campus, principalmente no RU do refeitório II, conforme mostra a Figura 5. 
Figura 5 - RU mais frequentado X (Almoçar em grupo ou sozinho)

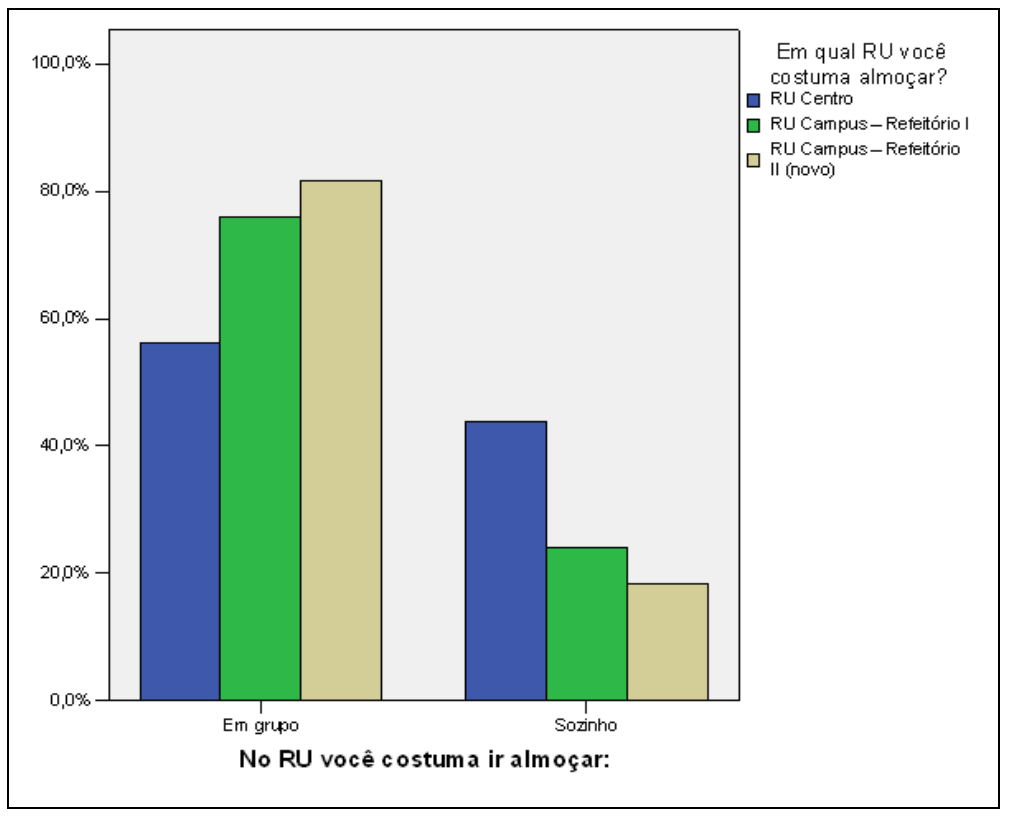

Ao analisarmos a faixa etária dos usuários do Restaurante Universitário representada pela Figura 6, notamos que 71,86\% são jovens com idade entre 17 e 25 .

Figura 6 - Histograma da faixa etária dos usuários do RU - UFSM

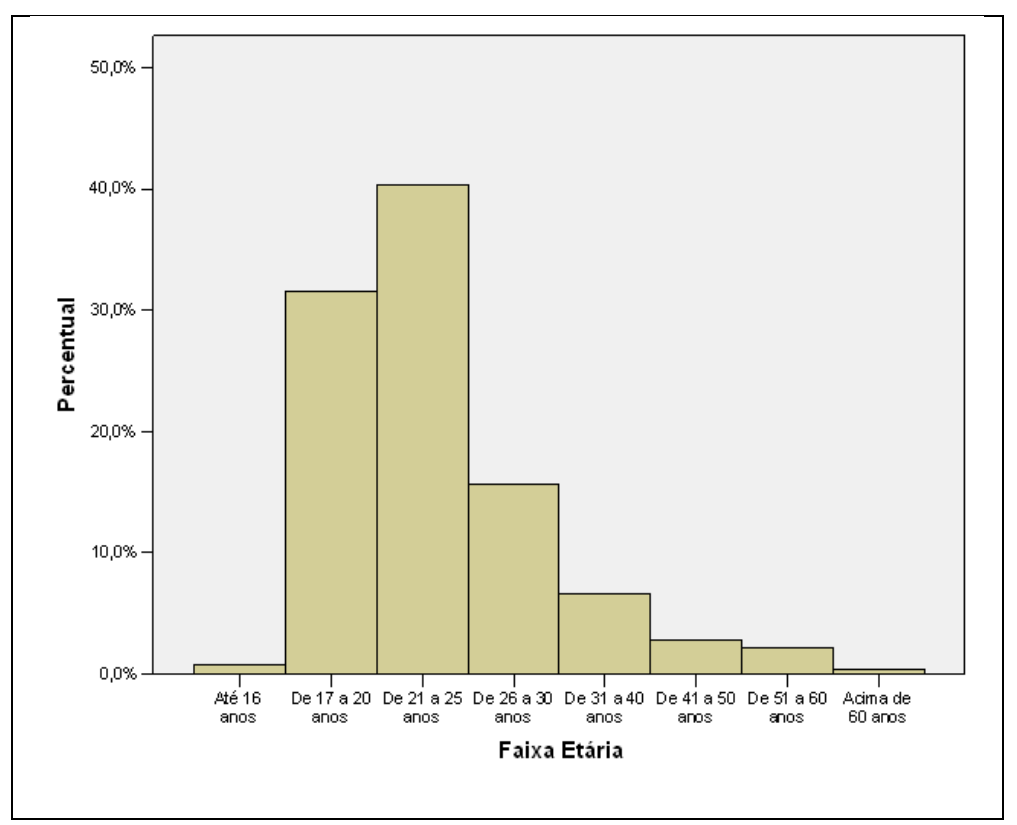

Dentre os frequentadores do RU, 34,5\% almoçam antes do meio-dia, já 58,44\% preferem fazer esta refeição no intervalo do meio-dia às 13 horas, restando uma pequena parcela de 7,06\% que opta por almoçar após as 13 horas. Com relação à frequência, $44,47 \%$ dos usuários almoçam diariamente no RU, 31,54\% de 3 a 4 vezes 
por semana, 10,73\% duas vezes por semana, 1,89\% uma vez por semana e 11,37\% almoçam eventualmente no RU.

A maioria dos usuários optam pelo RU devido ao preço atrativo das refeições (58,3\%), outros $21,5 \%$ almoçam no RU por estar localizado próximo do local da aula, bolsa ou trabalho. Ainda, 7,1\% dos usuários escolhem o RU para almoçar devido à qualidade das refeições, 6,6\% devido à alimentação saudável proporcionada e 6,5\% devido a outros motivos.

A Tabela 4 apresenta a média dos indicadores de satisfação e importância, calculados segundo os valores atribuídos na escala likert, para cada atributo avaliado, além da média geral para cada grupo de atributos.

A média geral de todas as médias dos atributos de satisfação foi de 3,37, enquanto que para a importância foi de 4,23. A maior média de satisfação foi atribuída ao ambiente externo, enquanto que a maior média de importância foi atribuída ao atendimento.

O atributo com maior grau de satisfação médio foi o preço cobrado pelo RU $(4,21)$, seguido pela iluminação do restaurante $(4,08)$, localização próxima ao local das aulas $(3,97)$ e alimentação equilibrada e saudável $(3,81)$. O atributo com maior importância média atribuída foi a higiene dos alimentos (4,87), a qualidade dos alimentos $(4,84)$, a limpeza dos utensílios $(4,81)$ e a limpeza do ambiente interno $(4,79)$. A decoração $(2,62)$ e a Fachada/aparência externa $(2,86)$ apresentaram uma baixa importância ao usuário. Entre os itens avaliados com baixo grau de satisfação, destacam-se o tamanho da fila para entrar no $\mathrm{RU}(2,65)$, o tamanho da fila para a compra de créditos $(2,71)$ e a disponibilidade de lugar para sentar e almoçar $(2,76)$.

Dentre os 41 atributos analisados, 19 apresentaram satisfação média superior à média geral, o que representa 46,34\% dos atributos, e 27 apresentaram importância média superior à média geral, o que representa $65,85 \%$ dos atributos. 
Tabela 4 - Média dos indicadores de satisfação e importância para cada atributo

\begin{tabular}{|c|c|c|}
\hline Itens & Satisfação & Importância \\
\hline Ambiente interno & 3,36 & 4,07 \\
\hline Tamanho/estrutura & 3,49 & 4,29 \\
\hline Layout & 3,44 & 4,02 \\
\hline Limpeza & 3,79 & 4,79 \\
\hline Iluminação & 4,08 & 3,90 \\
\hline Odores/cheiros & 3,19 & 4,48 \\
\hline Ventilação & 3,11 & 4,69 \\
\hline Conforto & 3,61 & 3,98 \\
\hline Barulhos & 2,82 & 3,66 \\
\hline Decoração & 3,18 & 2,62 \\
\hline Banheiro & 2,83 & 4,31 \\
\hline Ambiente externo & 3,42 & 3,90 \\
\hline Fachada/aparência & 3,28 & 2,86 \\
\hline Localização & 3,97 & 4,04 \\
\hline Acessibilidade & 3,67 & 4,43 \\
\hline Abrigo do sol e chuva & 2,96 & 4,54 \\
\hline Sinalização & 3,23 & 3,64 \\
\hline Alimentação & 3,40 & 4,34 \\
\hline Organização do Buffet & 3,79 & 4,04 \\
\hline Qualidade dos alimentos & 3,60 & 4,84 \\
\hline Temperatura dos alimentos & 3,76 & 4,51 \\
\hline Equilibrada e saudável & 3,81 & 4,62 \\
\hline Sabor e temperos & 2,86 & 4,54 \\
\hline Higiene dos alimentos & 3,67 & 4,87 \\
\hline Variedade diária & 3,25 & 4,34 \\
\hline Variedade do cardápio semanal & 3,19 & 4,35 \\
\hline Quantidade da porção da carne & 3,29 & 3,99 \\
\hline Quantidade da porção sobremesa & 3,45 & 3,62 \\
\hline Salada & 3,23 & 4,38 \\
\hline Arroz & 3,70 & 4,34 \\
\hline Complemento & 3,27 & 4,37 \\
\hline Feijão/lentilha & 3,46 & 4,45 \\
\hline Carne & 3,10 & 4,48 \\
\hline Guisado de soja & 2,94 & 3,63 \\
\hline Atendimento & 3,31 & 4,40 \\
\hline Apresentação dos funcionários & 3,72 & 4,28 \\
\hline Agilidade em repor os alimentos & 3,53 & 4,49 \\
\hline Manutenção do cardápio & 3,16 & 4,37 \\
\hline Preço cobrado & 4,21 & 4,55 \\
\hline Opções de pagamento & 3,65 & 4,01 \\
\hline Horário para a compra de créditos & 3,50 & 4,17 \\
\hline Tamanho da fila para a compra de créditos & 2,70 & 4,29 \\
\hline Tamanho da fila para entrar no RU & 2,65 & 4,47 \\
\hline Disponibilidade de lugar para almoçar & 2,76 & 4,51 \\
\hline Limpeza dos utensílios & 3,21 & 4,81 \\
\hline
\end{tabular}


A Figura 7 apresenta a Janela do Cliente para os atributos avaliados nessa pesquisa. Os eixos da satisfação e da importância estão apresentados com o intervalo entre 1 e 5, determinados pelos valores da escala likert, conforme sugerido por Albrecht e Bradford (1992), no entanto a "zona cinzenta" foi desconsiderada nesse estudo. As médias da satisfação e da importância delimitaram os quadrantes no gráfico.

Figura 7 - Janela do Cliente aplicada aos atributos do RU

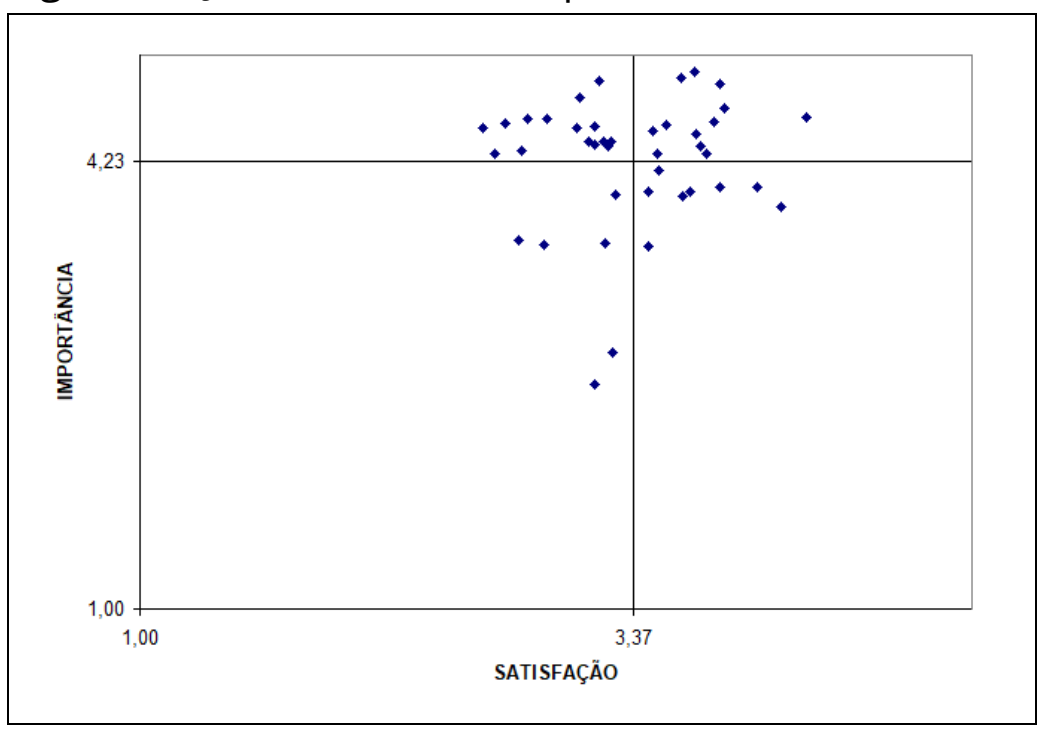

Como foi mencionado anteriormente, utilizar as médias da satisfação e da importância para delimitar os quadrantes não parece ser uma prática justa, embora seja muito utilizada. Observa-se, por exemplo, que o atributo "sinalização", embora tenha satisfação média de 3,23 (acima de indiferente) e importância média de 3,64 (entre razoavelmente importante e importante), foi situado no quadrante C (Relativa Indiferença), onde os usuários não valorizam. Outro exemplo de incoerência é o atributo "localização" que teve satisfação média de 3,97 (praticamente satisfeito) e importância média de 4,04 (acima de importante), mas foi classificado no quadrante B (Superioridade Irrelevante), onde o cliente está satisfeito, mas não dá importância. Por causa desses resultados incoerentes, o valor delimitante para os quadrantes adotado nesse trabalho foi o valor 3, que representa o meio termo na escala likert entre 1 e 5. Assim, a Figura 8 apresenta a Janela do Cliente para os atributos avaliados nessa pesquisa, com os quadrantes delimitados pelo valor 3. 
Por meio da Figura 8, podemos verificar que os atributos "sinalização" e "localização" do RU agora estão posicionados no Quadrante A (Força Competitiva), ou seja, os usuários do RU dão importância e estão satisfeitos com esses atributos. Os atributos "decoração" e "fachada/aparência" estão situados no Quadrante B (Superioridade Irrelevante), ou seja, os usuários do RU estão satisfeitos com a decoração interna e com a aparência externa, porém isso não é importante para eles. Os itens críticos, que estão situados no Quadrante D (Vulnerabilidade Competitiva) são "abrigo do sol e chuva", "sabor e temperos", "temperatura dos alimentos", "tamanho da fila da entrada", "tamanho da fila para a compra de créditos", "banheiro", "barulhos" e "guisado de soja". São esses os fatores que podem incentivar os usuários a procurar outro local para almoçar. Os demais atributos encontram-se no Quadrante A (Força Competitiva), ou seja, são importantes e foram bem avaliados. Nenhum atributo ficou situado no Quadrante C (Relativa Indiferença).

Figura 8 - Janela do Cliente aplicada aos atributos do RU com valor delimitante 3 nas escalas

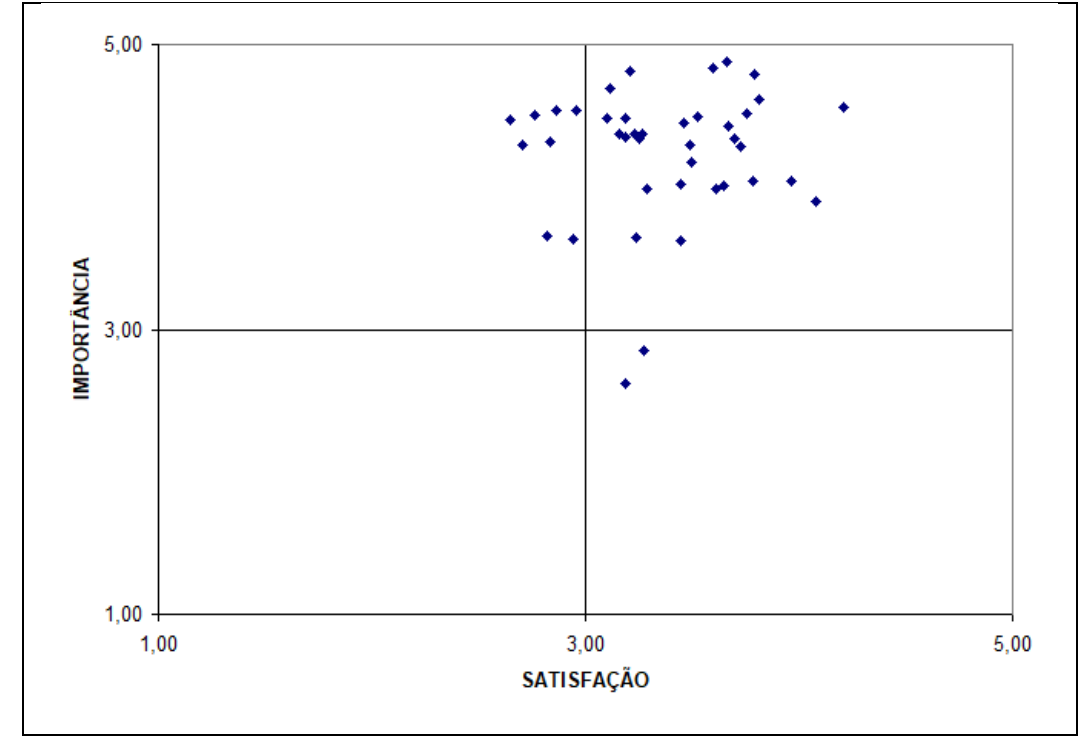

Com o intuito de verificar os itens vulneráveis de cada restaurante individualmente, a ferramenta Janela do Cliente foi aplicada para cada uma das três unidades. A Figura 9 apresenta a Janela do Cliente para os atributos avaliados no Restaurante do Centro (RU-C). 
Figura 9 - Janela do Cliente aplicada aos atributos do RU - Centro

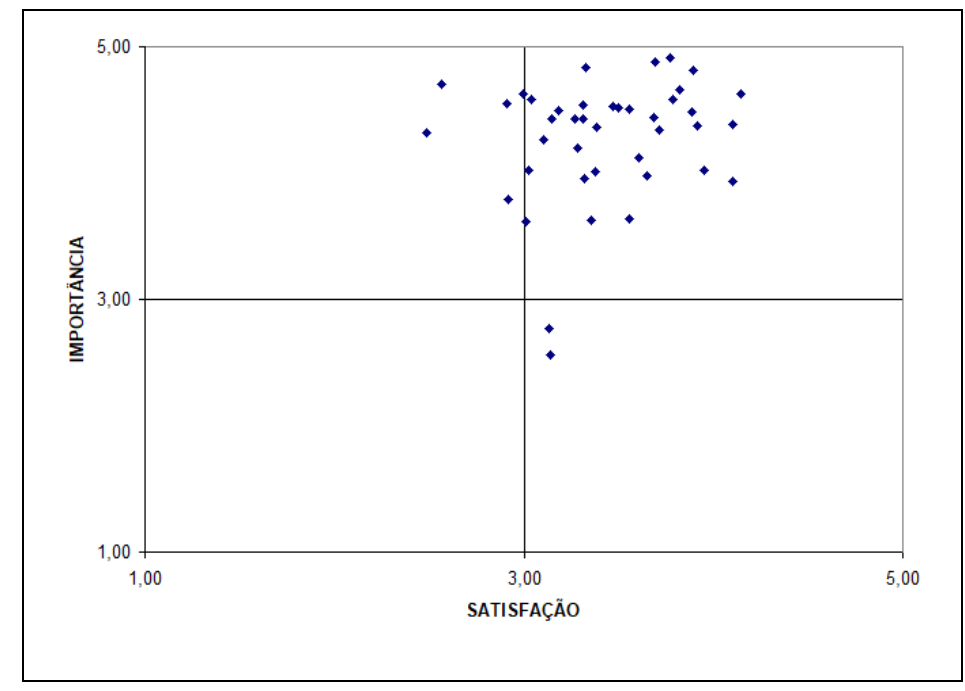

Concordando com o resultado da análise conjunta da Figura 8, os atributos "decoração" e "fachada/aparência" estão situados no Quadrante B (Superioridade Irrelevante), ou seja, os usuários do RU do Centro estão satisfeitos com a decoração interna e com a aparência externa, porém isso não é importante para eles. Os itens críticos, que estão situados no Quadrante D (Vulnerabilidade Competitiva) foram "ventilação", "sabor e temperos", "banheiro", "guisado de soja" e "disponibilidade de lugar para sentar". São esses os fatores que podem incentivar os usuários a procurar outro local para almoçar. Os atributos restantes encontram-se no Quadrante A (Força Competitiva) e nenhum atributo ficou situado no Quadrante C (Relativa Indiferença).

A Figura 10 apresenta a Janela do Cliente para os atributos avaliados no Restaurante do Campus, no Refeitório I (RU-I).

Figura 10 - Janela do Cliente aplicada aos atributos do RU do Campus - Refeitório I

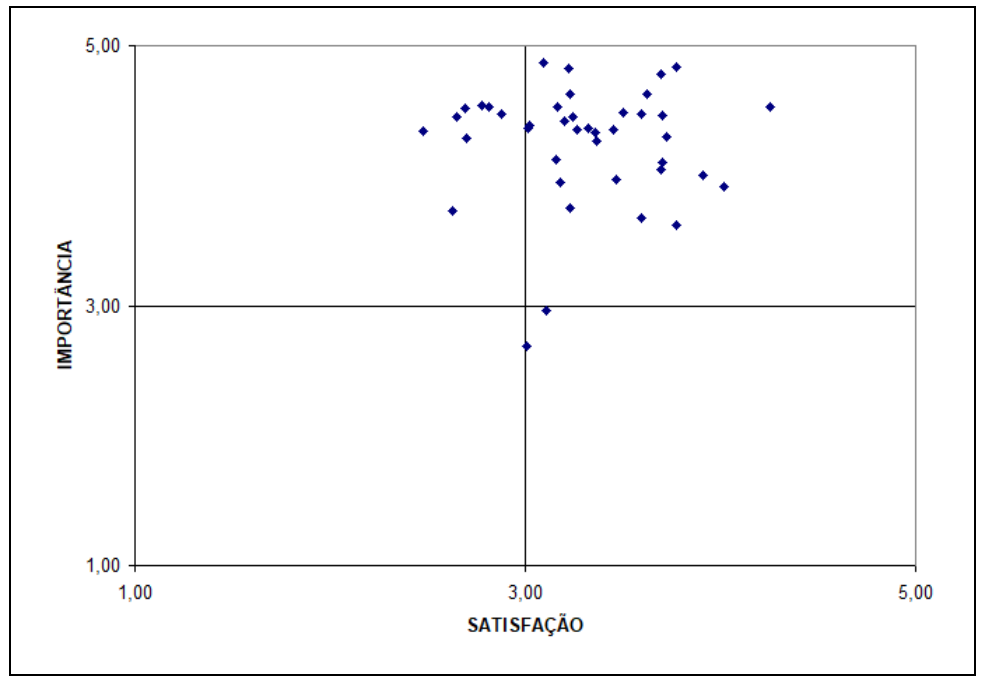


Assim como no resultado do Restaurante do Centro, os atributos "decoração" e "fachada/aparência" estão situados no Quadrante B (Superioridade Irrelevante), ou seja, os usuários do RU do Campus, no Refeitório I, estão satisfeitos com a decoração interna e com a aparência externa, porém isso não é importante para eles. Os itens críticos, situados no Quadrante D (Vulnerabilidade Competitiva) foram "feijão/lentilha", "odores/cheiros", "temperatura dos alimentos", "disponibilidade de lugar para sentar", "tamanho da fila da entrada", "tamanho da fila para a compra de créditos", "banheiro" e "barulhos". Os resultados para o Quadrante A e C foram os mesmos com relação ao $\mathrm{RU}$ - Centro.

A Figura 11 apresenta a Janela do Cliente para os atributos avaliados no Restaurante do Campus, no Refeitório II (RU-II).

Figura 11 - Janela do Cliente aplicada aos atributos do RU do Campus - Refeitório II

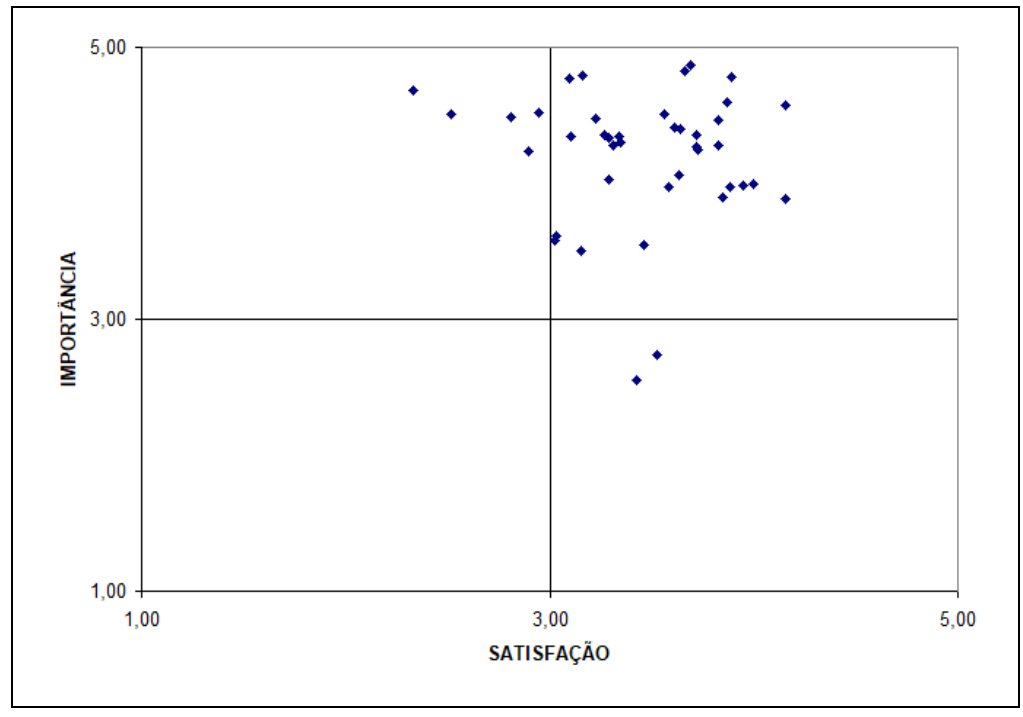

Assim como no resultado do Restaurante do Centro e do Refeitório I, os atributos "decoração" e "fachada/aparência" se mostraram situados no Quadrante B (Superioridade Irrelevante). Os itens críticos, situados no Quadrante D (Vulnerabilidade Competitiva) foram "disponibilidade de lugar para sentar", "sabor e temperos", "tamanho da fila da entrada", "tamanho da fila para a compra de créditos", "abrigo do sol e da chuva". Para os Quadrantes A e C os resultados foram análogos ao RU - Centro e RU do Campus - Refeitório I. 
O Quadro 1 apresenta um resumo com os itens considerados críticos em cada unidade do Restaurante Universitário. Esses itens são importantes para os usuários, porém não estão funcionando de forma satisfatória.

Quadro 1 - Itens críticos para cada unidade do Restaurante Universitário

\begin{tabular}{|l|c|c|c|}
\hline \multirow{2}{*}{ Atributos } & \multicolumn{3}{|c|}{ Unidade do Restaurante Universitário } \\
\cline { 2 - 4 } & Centro & Refeitório I & Refeitório II \\
\hline Disponibilidade de lugar para sentar & $\mathrm{X}$ & $\mathrm{X}$ & $\mathrm{X}$ \\
Banheiros & $\mathrm{X}$ & & $\mathrm{X}$ \\
Sabor e temperos & $\mathrm{X}$ & & \\
Ventilação & $\mathrm{X}$ & $\mathrm{X}$ & $\mathrm{X}$ \\
Guisado de soja & $\mathrm{X}$ & $\mathrm{X}$ & $\mathrm{X}$ \\
Tamanho da fila da entrada & & $\mathrm{X}$ & \\
Tamanho da fila para a compra de & & $\mathrm{X}$ & \\
créditos & & $\mathrm{X}$ & \\
Odores/cheiros & & $\mathrm{X}$ & \\
Temperatura dos alimentos & & & $\mathrm{X}$ \\
Barulhos & & \\
Feijão/lentilha & & \\
Abrigo do sol e da chuva & & \\
\hline
\end{tabular}

Conforme observado no Quadro 1, a "disponibilidade para sentar" após se servir com a refeição é um problema comum nas três unidades dos RU's. Os usuários, após se servir, têm dificuldade em encontrar um lugar vazio para sentar e almoçar, principalmente quando eles vêm almoçar em grupo de até quatro alunos e não encontram uma mesa que tenha lugar para todos. Isso reflete um problema de lotação nos RU's, que teve grande contribuição do aumento do número de Cursos e de vagas na UFSM nos últimos anos. As condições gerais dos banheiros têm sido um problema para os RU's do Centro e no Refeitório I. Os usuários têm se queixado das condições de limpeza e higiene, da conservação física (torneiras que não funcionam e tampas de vaso sanitário quebradas) e da falta de sabonete e de papel higiênico. O sabor e o tempero dos alimentos têm sido uma queixa nos RU's do Centro e no Refeitório II, onde a diminuição do sal na comida e a indisponibilidade do saleiro têm sido as principais queixas. Os RU's do Campus têm problemas com as longas filas para entrar no RU e para comprar créditos. Fato esse que se justifica pelo aumento de Cursos no Campus, pois além do aumento de Cursos na UFSM, vários cursos que antes se situavam no Centro da cidade foram transferidos para o Campus, e outros ainda serão transferidos. 
Apesar de existirem problemas em comum entre os RU's, a maioria dos atributos críticos é específica de cada restaurante. O RU do Centro apresenta problemas de ventilação, pois se situa no andar térreo de um prédio da UFSM e possui poucas janelas. Os usuários também não estão satisfeitos com o guisado de soja no RU do Centro. O Refeitório I apresenta vários problemas. Há muitas críticas dos usuários com relação aos odores e cheiros principalmente na área da devolução dos utensílios, próximo à porta de saída do refeitório. Os barulhos no ambiente deste RU também incomodam os usuários. Além disso, os usuários demonstraram-se insatisfeitos com a temperatura dos alimentos e com o feijão ou a lentilha. O Refeitório II se destaca por apresentar um problema externo em relação ao abrigo do sol e da chuva, que é insuficiente para o tamanho da fila na entrada do RU. Esses fatores climáticos são típicos da Cidade de Santa Maria, principalmente o calor em época de verão, que ultrapassa os 40 graus Celsius, tornando insuportável a espera em uma fila debaixo do sol.

A Tabela 5 apresenta um resumo da quantidade e do percentual de itens que estão situados em cada Quadrante, no conjunto e para cada restaurante.

Tabela 5 - Quantidade (e percentual) de itens em cada Quadrante, por Restaurante

\begin{tabular}{lcccc}
\hline Quadrante/Restaurante & Conjunto & RU Centro & RU I Campus & RU II Campus \\
\hline Quadrante A & $31(75,6 \%)$ & $34(82,9 \%)$ & $31(75,6 \%)$ & $34(82,9 \%)$ \\
Quadrante B & $2(4,9 \%)$ & $2(4,9 \%)$ & $2(4,9 \%)$ & $2(4,9 \%)$ \\
Quadrante C & $0(0.0 \%)$ & $0(0.0 \%)$ & $0(0.0 \%)$ & $0(0.0 \%)$ \\
Quadrante D & $8(19,5 \%)$ & $5(12,2 \%)$ & $8(19,5 \%)$ & $5(12,2 \%)$ \\
\hline
\end{tabular}

Observa-se que o Refeitório I foi o que apresentou a maior quantidade de itens $(19,5 \%)$ no Quadrante D (Vulnerabilidade Competitiva), enquanto que os demais restaurantes apresentaram $12,2 \%$ dos itens neste quadrante. Todos os restaurantes apresentaram apenas 2 itens (4,9\%) no Quadrante B (Superioridade Irrelevante), que, por sinal, foram os mesmos itens em todos eles. No entanto, a maioria dos itens encontram-se em conformidade, no Quadrante A (Força Competitiva), sendo 82,9\% dos itens do RU Centro e do Refeitório II, e 75,6\% no Refeitório I. Nenhum restaurante teve item situado no Quadrante C (Relativa Indiferença). 


\section{CONSIDERAÇÕES FINAIS}

O objetivo deste trabalho foi apresentar uma análise crítica da aplicação do método da ferramenta conhecida como Janela do Cliente na avaliação da qualidade do Restaurante Universitário da Universidade Federal de Santa Maria - RS (UFSM). No entanto, no estudo da literatura disponível sobre a ferramenta Janela do Cliente, observou-se que os pesquisadores têm determinado o cruzamento dos eixos, para a identificação dos quadrantes, por meio da média da satisfação e da importância de todos os atributos, avaliados em uma escala likert de cinco pontos. Segundo essa abordagem, por mais bem avaliados que os atributos sejam, sempre haverá alguns acima da média e outros abaixo da média, ou seja, sempre haverá atributos em pelo menos dois quadrantes, o que torna essa abordagem incoerente. Desta forma, optouse por utilizar os valores médios das escalas, no caso, o valor 3 na escala likert de 1 e 5 , para delimitar os quadrantes.

Os resultados mostraram que a maioria dos itens encontram-se em conformidade, no Quadrante A, sendo 82,9\% dos itens do RU Centro e do Refeitório II, e $75,6 \%$ no Refeitório I. Além disso, todos os restaurantes apresentaram os mesmos dois itens $(4,9 \%)$ no Quadrante B e nenhum item situado no Quadrante C. No entanto, o Refeitório I foi o que apresentou a maior quantidade de itens (19,5\%) no Quadrante $D$, enquanto que os demais restaurantes apresentaram $12,2 \%$ dos itens nesse quadrante. Apesar de existirem problemas em comum entre os RU's, foi identificado que a maioria dos atributos críticos é específica de cada restaurante, sendo que o único problema comum nas três unidades dos RU's é a dificuldade em encontrar um lugar vazio para sentar e almoçar, após o usuário se servir com a refeição, principalmente quando eles vêm almoçar em grupo.

A Janela do Cliente, segundo a abordagem utilizada nesse trabalho, se mostrou uma ferramenta simples e eficiente, capaz de identificar os pontos falhos e fortes do Restaurante Universitário. Os resultados dessa pesquisa foram encaminhados à administração do Restaurante Universitário da UFSM, onde poderão gerar subsídios para formulação de propostas que visam melhorias (mudanças ou adaptações) nos atributos avaliados. 


\section{REFERÊNCIAS}

AAKER DA, KUMAR V, DAY GS. Pesquisa de Marketing. São Paulo: Atlas; 2001.

ALBRECHT K, BRADFORD LJ. Serviços com qualidade: a vantagem competitiva. São Paulo: Makron Books; 1992.

ASSESSORIA DE PESQUISA E DESENVOLVIMENTO GERENCIAL. Customer Window: catálogo de informações. São Paulo: Rhodia S.A.; 1994.

BARROSO FR. Fatores de localização de empresas de tecnologia da informação em Parques Tecnológicos do Estado do Rio Grande do Sul [dissertation]. Porto Alegre: Programa de Pós-Graduação em Administração/Universidade Federal do Rio Grande do Sul; 2007.

BRANDÃO AR, GIOVANONI A. Comparação dos cardápios oferecidos em uma unidade de nutrição do município de Teutônia com o programa de alimentação do trabalhador. Revista Destaques Acadêmicos. 2011;3(3).

CÁCERES LBA, Janela do Cliente [monography]. Porto Alegre: Escola de Administração. Universidade Federal do Rio Grande do Sul; 1994.

CARVALHO LR, AMORIM SL, TAVARES MF. Sistema de indicadores de qualidade como ferramenta de gestão em um restaurante universitário: estudo de caso. In: XXIII Encontro Nacional de Engenharia de Produção; 2003 October 21-24; Ouro Preto; MG; 2003.

FEOLI CFR. Pesquisa de satisfação dos clientes da Hiperacademia. [monography]. Porto Alegre: Escola de Administração. Universidade Federal do Rio Grande do Sul; 2006.

FONAPRACE [Internet]. Realidade dos RUs em 1997 [cited 2013 jun 24]. Perfil dos Restaurantes Universitários das Instituições de Ensino Superior do País, 1997. Available from: http://www.ufrn.br/sites/fonaprace/rus.html.

GARCEZ EMS, FACHIN GRB, ANDRADE JÚNIOR PP. Indicadores da qualidade em restaurantes: um estudo de caso. Revista de Ciências da Administração. 2000;2(3):29-38.

HALEY RI. Developing effective communications strategy: A benefit segmentation. New York: John Wiley \& Sons; 1985.

KLASSEN K, KUMAR A, TRYBUS E. Planning food services for a campus setting. Internacional Journal of Hospitality Management, England. 2005;24(4):579-609. 
LUDWING ES. Janela do Cliente aplicada à Clínica Schuler. [monography]. Porto Alegre: Escola de Administração. Universidade Federal do Rio Grande do Sul; 2008.

MAIA TML. Planejamento e gestão estratégica para o restaurante universitário da UFC em um cenário de expansão do número de alunos [dissertation]. Ceará: Universidade Federal do Ceará; 2008.

MARTILLA JA, JAMES JC. Importance-performance analysis. Journal of marketing. 1977:41.

MATTOS AP. Pesquisa de satisfação de clientes na perspectiva do modelo "janela do cliente". Caso: Megaset assessoria de seguros Ltda. Porto Alegre - RS [monography]. Gravataí: Curso de Administração. Universidade Luterana do Brasil; 2008.

MCDANIEL CD, GATES R. Pesquisa de marketing. São Paulo: Thomson Learning; 2006.

NOTARI JN. A satisfação de clientes em uma loja autocentro de pneus através do enfoque da "janela do cliente" [dissertation]. Porto Alegre: Programa de PósGraduação em Administração/Universidade Federal do Rio Grande do Sul; 2002.

OLIVEIRA CS, ALVES FS. Educação nutricional em unidade de nutrição, direcionada para consumo de pratos protéicos um estudo de caso. Nutricional. 2008;19(4):435-440.

PASSADOR JL, FILHO DOL, SPANHOL PS, RODRIGUES FS, SABES JJS. A percepção do consumidor de alimentos "fora de casa": um estudo multicaso na cidade de Campo Grande/MS. In: XIII SIMPEP; 2006 nov 6-8; Bauru, SP, Brasil.

PASTORE JA, KLIEMANN N, FERNANDES AC, OLIVEIRA RC, CORSO ACT, PROENÇA RPC. A opinião dos comensais sobre a refeição servida em uma Unidade de Alimentação e Nutrição sob a ótica do saudável. Nutrição em Pauta; 2009; 17:54-58.

PORTAL WH. Pesquisa de satisfação com consumidores da Pizzaria Portal, com a utilização da Janela do Cliente [monography]. Porto Alegre: Escola de Administração. Universidade Federal do Rio Grande do Sul; 2012.

PROENÇA RPC. Novas tecnologias para a produção de refeições coletivas: recomendações de introdução para a realidade brasileira. Rev. Nutr. 1999;12(1).

ROSA, T. D. Pesquisa de satisfação e janela do cliente dos usuários do Estádio Beira-Rio [monography]. Porto Alegre: Escola de Administração. Universidade Federal do Rio Grande do Sul; 2011. 
ROSSI CAV, SLONGO LA. Pesquisa de Satisfação de clientes: o Estado-da-Arte e Preposição de um Método Brasileiro. Revista de Administração Contemporânea. 1998;2(1):101-125.

SCHLENDER HE. Estudo sobre a satisfação dos clientes da Loja Closet [monography]. Porto Alegre: Escola de Administração. Universidade Federal do Rio Grande do Sul; 2013.

SIEBENEICHLER T, WIENNINGKAMP D, RUCHEL AP, TROMBINI ES, ZAMBERLAN L. A Satisfação de Clientes de Restaurantes: Uma Avaliação da Satisfação e da Importância dos Atributos. In XI SemeAd; 2008 aug 28-29.

SILVA LA. Satisfação de clientes com processo de compra: Um estudo na empresa de telefonia móvel Telet S/A.[dissertation]. Porto Alegre: Programa de Pós-Graduação em Administração/Universidade Federal do Rio Grande do Sul; 2003.

SOUZA CHM. Uma ferramenta para a avaliação da qualidade em serviços - a Janela do Cliente. Perspectivas online. 2008;5(1):65-79.

TEIXEIRA S, MILLET Z, CARVALHO J, BISCONTINI TM. Administração aplicada às unidades de alimentação e nutrição. São Paulo: Atheneu; 2001.

ZABALETA NS. Janela do cliente do conjunto de serviços e produtos das lojas Varal [monography]. Porto Alegre: Escola de Administração. Universidade Federal do Rio Grande do Sul; 2011. 